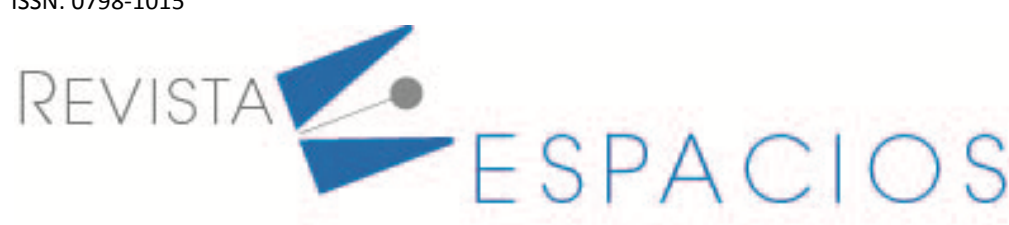

\title{
Modalidades de lectura de docentes de La Araucanía, Chile: ¿Qué podemos aprender de sus prácticas pedagógicas?
}

\section{Reading modalities of teachers from La Araucanía, Chile. What can we learn from their teaching practices?}

\author{
ERRÁZURIZ, María C. ${ }^{1}$ \\ DAVISON, Omar A. ${ }^{2}$ \\ COCIO, Andrea C. ${ }^{3}$ \\ FUENTES, Liliana I. ${ }^{4}$
}

\begin{abstract}
Resumen
Como las prácticas pedagógicas para desarrollar la lectura son fundamentales para el logro de esta, el objetivo fue analizar las modalidades de lectura en las prácticas docentes en escuelas públicas de La Araucanía, Chile. Para ello se videograbaron 1.485 minutos de clases 11 profesoras y se analizaron sus episodios de modalidades de lectura. Entre los resultados se destaca que la lectura compartida fue la más frecuente y que no se explicitan los beneficios de las distintas modalidades de lectura.

Palabras clave: prácticas docentes, modalidades de lectura, lectura

Abstract

As the teaching practices to develop reading are essential to achieve them, the objective was to analyze the reading modalities in pedagogical practices in public schools of La Araucanía, Chile. In order to do this, 1,485 minutes of classes were videotaped, 11 teachers and their episodes of reading modalities were analyzed. Among the results, shared reading was highlighted as the most frequent and the benefits of the different reading modalities were not explicit.

key words: teaching practices, reading, reading modes
\end{abstract}

\section{Introducción}

La efectividad de las prácticas pedagógicas del profesorado constituye uno de los factores claves para la calidad y el nivel de inclusión de los aprendizajes de los estudiantes y, por tanto, de la educación escolar (Barber y Mourshed, 2008; Liston, Borko \& Withcomb, 2008). No obstante, según la evidencia disponible en Chile, las prácticas docentes, en general, son mayoritariamente reproductivas, con presencia de preguntas cerradas y el dominio del discurso docente (Molina-Valdés y Haas-Prieto, 2018; Manzi y García, 2016; Preiss et al., 2014). Esta misma situación también se ha observado en las prácticas de mediación de la lectura en el aula escolar, pues también en ellas predominan la formulación de preguntas de mediano a bajo desafío cognitivo y patrones de interacción más bien directivos y monologales (Bustos-Ibarra, Montenegro, Jarpa-Azagra, Calfual-Catalán y

\footnotetext{
${ }^{1}$ Profesora Asociada. Campus Villarrica. Pontificia Universidad Católica de Chile. cerrazuc@uc.cl

${ }^{2}$ Coordinador de Programas Educativos en Huiscapi. Coordinación Huiscapi. Fundación Ibáñez-Atkison. omardavisont@gmail.com

${ }^{3}$ Profesora Asistente. Facultad de Educación. Universidad Católica de Temuco. acocio@uct.cl

${ }^{4}$ Profesora Asociada. Facultad de Filosofía y Humanidades. Universidad de Chile. lilianafuentes@uchile.cl
} 
Tapia-Ibacache, 2019; Iturra, 2014), en donde el docente tiene total control del plan de acción y lo comparte en pocos momentos.

Lo anterior implicaría que se estarían desplegando modos únicos de solucionar problemas, lo que está conectado con una representación de la enseñanza como reproducción (Alexander, 2008), por lo que se continúa manteniendo la inequidad del sistema (Valenzuela, Bellei \& Ríos, 2014). Especialmente, si consideramos que la práctica frecuente de interacciones dialógicas en el contexto escolar ha demostrado desarrollar aprendizajes profundos, el habla exploratoria, la argumentación, el trabajo colaborativo y el interpensar de manera conjunta (Alexander, 2008; Mercer, 2001; Wells y Barberán, 2001). Más aún en las actividades de lectura, donde métodos y estrategias de interacción dialógica como la discusión productiva han sido exitosos para desarrollar el fomento lector, mediar la comprensión e interpretación de los textos y la construcción colectiva de sentidos (Kucan \& Palincsar, 2013; Larraín, Howe y Cerda, 2014; Meneses, Müller, Hugo y García, 2018).

De alguna manera, esto podría explicar por qué no hay avances significativos en las competencias y prácticas de lectura de los estudiantes del sistema escolar en Chile, expresadas por evaluaciones como PISA 2015 y PIRLS 2016 e investigaciones (Errázuriz et al., 2020; Errázuriz, Becerra, Cocio, Davison y Fuentes, 2018; OECD, 2016). De hecho, se ha constatado que a medida que el alumnado avanza de grado, las actitudes positivas hacia la lectura van decayendo junto con su rendimiento lector y que existe una brecha de género a favor de las niñas (Errázuriz et al., 2018; Latorre, 2014; Orellana \& Baldwin, 2018). Asimismo, existen evidencias sobre las dificultades y bajo rendimiento que tienen en literacidad los estudiantes de programas de formación inicial docente (Errázuriz, 2019; Muñoz \& Valenzuela, 2020).

Como sabemos, el desarrollo de la lectura es central para la educación escolar porque es indispensable para el aprendizaje en todas las disciplinas al ser una herramienta de mediación del conocimiento (Errázuriz, 2019, 2017; Bazerman et al., 2012; Carlino, 2005; Tolchinsky y Simó, 2001; Street \& Street, 1995) y al permitirnos conformar comunidades de práctica (Shanahan \& Shanahan, 2012). A lo anterior, se añaden los efectos cognitivos beneficiosos de la cantidad de lectura en la mente de los individuos (Cunningham \& Stanovich, 1998) y que es indispensable para la formación de ciudadanos responsables en sociedades democráticas y sostenibles (Unesco, 2017; Cassany, 2006) y para la emancipación de las personas (Freire, 2012), como lo expresa el Plan Nacional de la Lectura chileno, al señalar que es un derecho social (Consejo Nacional de la Cultura y las Artes, 2015).

A partir de lo anterior, el objetivo del presente estudio ha sido analizar las modalidades de lectura presentes en las prácticas pedagógicas de desarrollo de la lectura de profesoras de distintas disciplinas de escuelas públicas de la Región de La Araucanía, Chile. De este modo, esperamos contribuir comprender con mayor profundidad estas prácticas y, así, potenciarlas, dado que la manera en como modelamos y mediamos la lectura puede tener un gran impacto en las prácticas lectoras de los estudiantes.

\section{Marco teórico}

\subsection{La mediación de la lectura en las disciplinas}

Como la comprensión de las lecturas de los alumnos se acuerda en comunidad, es el docente quien, como lector experto, debe traspasar progresivamente su experiencia y el monitoreo al lector novato (Sánchez, García, Rosales, 2010) y, por ello, media los textos y configura un espacio de referencias en común (Chartier y Hébrard, 2000). De hecho, el modelo de Rosenblatt (1985), define la lectura como un proceso de interacción entre el lector y el texto, de acuerdo con los propósitos que orienten la lectura, lo que permite construir un significado, por ello un lector más activo puede construir una comprensión más profunda y adecuada, según su objetivo. No obstante, según Rockwell (2001), hay prácticas de enseñanza de la lectura que distancian a los estudiantes de la cultura escrita y que los excluye, al no mediar los textos y relacionarlos con su experiencia y no orientar un sentido. 
Según algunos estudios, esto sucede porque se ha observado que los estudiantes del sistema escolar y de pedagogía presentan propósitos de lectura principalmente pragmáticos, es decir, leen solo para la evaluación, pues no lo hacen frecuentemente ni efectúan una interpretación personal de los textos (Muñoz et al., 2016).

En este sentido, se ha identificado la relación que existe entre las concepciones y hábitos de literacidad del profesorado, sus prácticas de enseñanza y mediación lectora y el modelamiento de la motivación en literacidad del alumnado (Errázuriz et al., 2019; Benevides \& Peterson, 2010; Morrison, Jacobs \& Swinyard, 1999; Muñoz, Munita, Valenzuela y Riquelme, 2018). En efecto, se ha observado que el profesorado escolar chileno que presenta perfiles lectores más epistémicos asocia las prácticas de lectura con el placer, media la lectura de sus estudiantes, se interesa por motivarla y son más inclusivos (Errázuriz et al., 2019; Errázuriz et al., 2018). Sin embargo, sabemos que aún existe una resistencia por parte de los profesores de otras asignaturas respecto de mediar la lectura, pues creen que es una práctica propia de la escuera de la lengua (Muñoz et al., 2016; Carlino, 2005) y no necesariamente una actividad habitual en todas las disciplinas; por tanto, predomina un enfoque generalista y poco contextualizada de la lectura (Shanahan \& Shanahan, 2012).

Desde otros puntos de vista, se ha afirmado que los alumnos sí leen y sostienen sus propias prácticas de lectura, no obstante, en muchos casos no son abordadas en el aula escolar (Winocur, 2015). En consecuencia, de acuerdo con Zavala (2011), es necesario también validar las prácticas de lectura híbridas de los estudiantes y que los docentes integren los fondos de conocimientos de su alumnado (Llopart \& Esteban-Guitart, 2018) y construya comunitariamente los sentidos de los textos. De este modo, será posible explicitar las expectativas implícitas sobre la literacidad (Zavala, 2011) y, así, articular un proceso de mediación más inclusivo.

\subsection{Modalidades de lectura en las prácticas pedagógicas}

Las modalidades de lectura del profesor y su modelamiento en los estudiantes es uno de los factores que contribuye a mediar, andamiar y motivar la lectura en el aula. Con respecto a las modalidades de lectura, Swartz (2010) distingue las siguientes:

Lectura en voz alta: consiste en que el maestro le lee a los estudiantes para motivarlos y cubrir las necesidades de estudiantes con distintos niveles lectores. Para el lector experto, esta instancia constituye una oportunidad de interacción para compartir lo que entienden y construir relaciones. Asimismo, el docente, al modelar la lectura, se vuelve un modelo sobre cómo los buenos lectores leen y facilita el disfrute de aquellos estudiantes que aún presentan dificultades de decodificación. Algunos de los benficios de esta modalidad de lectura son facilitar aprendizaje de lectura, familiarizarse con distintos géneros discursivos y estrategias, incrementar el léxico, ayudar a comprender el funcionamiento de la lengua escrita y ampliar las habilidades cognitivas. En este sentido, según Hoffman (2011), la lectura en voz alta puede fomentar el desarrollo del lenguaje por medio del diálogo que se suscite con la lectura, siempre que se practique habitualmente y las actividades durante el proceso sean conscientemente programadas (Otto, 2008). Ahora bien, de acuerdo con Dickinson (2001), esta modalidad genera mayores aprendizajes cuando se motiva al alumnado a participar en la interacción, formulando interrogantes y promoviendo las predicciones, por ello es necesario que el docente andamie procesos de pensamiento de alto nivel (McGee y Schickedanz, 2007).

Lectura compartida: consiste en que el profesor y los estudiantes leen juntos, sucediéndose alternadamente los turnos de lectura, por lo que estos últimos pueden ver y seguir el texto. Otra manera de llevar a cabo esta modalidad es a través de la lectura en conjunto simultánea de todos los estudiantes y el maestro al unísono y, general, es frecuente en los primeros años de la educación escolar para promover la lectura inicial y la atención. En esta modalidad, el docente puede modelar las conductas lectoras y ofrecer diversos niveles de ayuda. En este sentido, esta instancia es una oportunidad para el logro de varios objetivos como dialogar sobre cómo entendemos lo que leemos, modelar la lectura fluida y expresiva, desarrollar el lenguaje oral y crear un clima de 
colaboración y comunidad. En efecto, según Larraín, Strasser y Lissi (2012), es una excelente instancia para definir y explicar el significado de las palabras con ejemplos sencillos y, así, desarrollar los aprendizajes.

Lectura guiada: consiste en que los estudiantes leen y el docente señala instrucciones explícitas de correspondencia fonema-grafema y para la comprensión. Esta modalidad es efectiva para modelar estrategias para formar lectores autónomos, incluir a alumnos que presentan necesitades educativas especiales y promover el aprendizaje cooperativo. Los beneficios que aporta son identificar y resolver dificultades en la comprensión del texto, reconocer y reflexionar sobre la estructura de la lengua, analizar palabras, desarrollar estrategias y comportamientos lectores y elevar los niveles de la lectura fluida y expresiva.

Lectura independiente: consiste en que los estudiantes asumen la responsabilidad de leer textos silenciosamente que escogen según sus preferencias, mientras el docente los observa, dialoga y monitorea. Esta modalidad contituye una oportunidad de practicar la lectura, de involucrarse en ella, desarrollar el léxico y las estrategias de comprensión textual y elegir de acuerdo con sus gustos. Su práctica debe ser progresiva, formular un objetivo de lectura y ofrecer la posibilidad de compartir preferencias e interpretaciones textuales.

A las modalidades ya descritas, incluimos la siguiente por su relevancia en el contexto escolar.

Lectura dramatizada: consiste en presentar y poner en relieve las diversas voces que emergen en el texto, bajo un enfoque más teatral; es decir, se apunta a una lectura que pueda "interpretar" el texto, en términos de destacar el componente expresivo y/o dramático según corresponda (Ministerio de Educación, 2013).

Asmismo, cabe destacar que en las distintas modalidades de lectura en voz alta dirigidas por parte del profesor o compartidas por todo el curso, el docente debe intencionar el lenguaje paraverbal y verbal para que motive y fomente la comprensión del texto. Por ejemplo, es importante que el maestro tome consciencia sobre su tono de voz, la entonación, el volumen, la postura, los gestos del rostro, las pausas, el dinamismo de estos elementos y la formulación de preguntas e ideas para desarrollar la interacción (Orellana-García, Valenzuela y Muñoz, 2018), para que así su lectura sea motivante y un modelo para los estudiantes.

A propósito de las modalidades descritas, según Gelber (2017), las actividades de lectura más frecuentes que los docentes efectúan en el contexto escolar son la lectura en voz alta y silenciosa, guías de trabajo sobre los textos leídos y el comentario en clases acerca de los textos abordados (Gelber, 2017). Asimismo, las actividades menos presentes son las dramatizaciones, la escritura creativa a partir de las lecturas y el análisis de textos. Irónicamente, las actividades que menos motivan al alumnado son desarrollar guías sobre las lecturas y escribir sobre los textos. En efecto, de acuerdo con una investigación de la Pontificia Universidad Católica de Chile (2011) en relación con las prácticas pedagógicas de alfabetización de primaria, la lectura en voz alta tanto del docente como de los estudiantes es una acción predominante con un 85,9\% de frecuencia.

Por útimo, respecto de los géneros discursivos presentes en estas modalidades de lectura en distintas asignaturas, según la evidencia nacional e internacional, se ha verificado que la dificultad para comprender géneros discursivos expositivos en el contexto escolar se debe posiblemente a la baja frecuencia de estos textos en las prácticas docentes, en constraste a la alta presencia de géneros narrativos (De Mier, Amado y Benítez, 2015; Errázuriz et al, 2020; Ruiz, Montenegro, Meneses y Venegas, 2016). Asimismo, es necesario considerar que estos textos presentan una mayor complejidad por su nivel de abstracción, su lenguaje especializado y su mayor manejo de de conocimientos disciplinares.

\section{Metodología}

La presente investigación se circunscribe como un estudio de casos múltiples (Flyvbjerg, 2006), descriptivo de corte cualitativo e interpretativo (Merriam \& Tisdell, 2009). 


\subsection{Participantes}

Las participantes corresponden a 11 profesoras de educación básica de 7 escuelas públicas de la Región de La Araucanía de Chile de tres ciudades (Temuco, Villarrica y Pucón), quienes fueron elegidas aleatoriamente y por disponibilidadad desde una muestra representativa de 338 docentes de 18 establecimientos educativos de las cuatro comunas más pobladas del territorio: Temuco, Villarrica, Angol y Pucón. Cabe señalar que esta región tiene los mayores índices de pobreza del país (Ministerio de Desarrollo Social, 2016) y una realidad multicultural que no ha sido suficientemente abordada (Becerra y Mayo, 2015).

Al momento del estudio, las participantes tenían un promedio de 20 años de experiencia en el aula y 7 de ellas contaban con estudios de posgrado. Asimismo, 10 de ellas son profesoras de Educación Básica y 1 es profesora de Ciencias Sociales; 3 son miembros de un pueblo originario, específicamente, mapuche y la totalidad realiza clases en distintas disciplinas de 3으 a 8 Básico. Algunos de los datos se especifican en la tabla 1, en donde los nombres de las docentes y sus escuelas corresponden a pseudónimos, para sí proteger sus identidades.

Tabla 1

Características de las docentes observadas

\begin{tabular}{|c|c|c|c|c|c|c|}
\hline Nombre & $\begin{array}{c}\text { Experiencia } \\
\text { (años de } \\
\text { servicio) }\end{array}$ & $\begin{array}{l}\text { Miembro } \\
\text { de pueblo } \\
\text { originario }\end{array}$ & Ciudad & $\begin{array}{c}\text { Título } \\
\text { profesional }\end{array}$ & Posgrado & Disciplina \\
\hline Pamela & 16 & no & Villarrica & $\begin{array}{c}\text { PEB } \\
\text { (Pedagogía en } \\
\text { Educación } \\
\text { Básica) }\end{array}$ & sí & Matemáticas \\
\hline Deborah & 6 & no & Temuco & PEB & sí & Lenguaje \\
\hline Magdalena & 25 & no & Villarrica & PEB & sí & Lenguaje \\
\hline Miriam & 20 & no & Villarrica & PEB & no & $\begin{array}{l}\text { Ciencias, } \\
\text { Lenguaje }\end{array}$ \\
\hline Monserrat & 20 & sí & Pucón & PEB & sí & Lenguaje \\
\hline Mariela & 36 & no & Pucón & PEB & sí & $\begin{array}{c}\text { Ciencias, } \\
\text { Lenguaje, } \\
\text { Matemáticas y } \\
\text { Tecnología }\end{array}$ \\
\hline Josefina & 20 & no & Villarrica & PEB & no & $\begin{array}{c}\text { Lenguaje, } \\
\text { Tecnología y } \\
\text { Artes }\end{array}$ \\
\hline Nicol & 18 & sí & Temuco & PEB & sí & Religión \\
\hline Nadia & 9 & sí & Temuco & PEB & & Matemáticas \\
\hline Magda & 30 & no & Villarrica & $\begin{array}{c}\text { Profesora de } \\
\text { Historia }\end{array}$ & sí & C. Sociales \\
\hline Elisa & 20 & no & Villarrica & PEB & no & $\begin{array}{c}\text { Lenguaje, } \\
\text { Ciencias }\end{array}$ \\
\hline
\end{tabular}

Fuente: elaboración propia

\subsection{Procedimientos}

A partir de lo anteriormente descrito, se videograbaron las prácticas docentes de las participantes ya mencionadas que presentaron actividades de lectura en distintas disciplinas. En particular, correspondieron a 3 sesiones de clases de 45 minutos de cada docente, es decir, 33 clases en total, lo que equivale a 1.485 minutos y 24,75 horas de clases lectivas. Además, se registraron notas de campo complementarias y se transcribió el audio de los videos. 
Con respecto al procedimiento de análisis, se siguió en parte la propuesta de Sánchez et al. (2010) desde un enfoque cualitativo; así, en primer lugar, se segmentaron las interacciones en dos unidades de análisis: ciclos y episodios. Los primeros corresponden a una unidad mínima compuesta por una serie de interacciones en torno al acuerdo entre dos o más individuos, mientras que los segundos se identifican como un conjunto de intercambios comunicativos entre profesores y estudiantes en relación al logro de un propósito común y están compuestos por varios ciclos.

En segundo lugar, se identificaron las modalidades de lectura empleados y los tipos de textos y géneros discursivos leídos en clases. Estas dimensiones fueron identificadas en el corpus por medio del programa Dedoose, calibradas por el equipo investigador y luego analizadas e interpretadas cualitativamente. En este sentido, se efectuó una codificación inicial para acordar y validar los primeros códigos. Posteriormente, en un segundo análisis por parte de los miembros del equipo, se llevó a cabo una codificación central para revisar y calibrar la codificación y establecer los códigos definitivos, con el fin de pasar a la fase de interpretación de los datos y codificación final. Además, durante los diferentes procesos de codificación se escribieron memos analíticos que fueron considerados en la interpretación de los resultados. En consecuencia, cada unidad identificada en el corpus se sometió al menos a tres revisiones y calibraciones por parte de distintos miembros del equipo investigativo.

\section{Resultados}

La experiencia de leer en el contexto del aula escolar puede variar según distintos factores como el tipo de texto empleado, la disciplina o el profesor. A continuación, presentamos los hallazgos que se derivan del análisis de la categoría modalidades de lectura que llevan a cabo 9 profesoras de la Región de la Araucanía en sus prácticas pedagógicas. De este modo, hemos identificado las siguientes seis modalidades de lectura: compartida alternada, compartida grupal y al unísono, en voz alta exclusiva del profesor, silenciosa, independiente y dramatizada.

En primer lugar, cabe señalar que la modalidad de lectura predominante fue la compartida alternada con 80 episodios, luego le sigue la lectura en voz alta del profesor con 37 episodios, posteriormente viene la lectura compartida al unísono con 13 episodios, luego le sigue la lectura silenciosa con 5 episodios y, por último, las modalidades minoritarias fueron la lectura independiente y la la lectura dramatizada con 3 episodios cada una. A continuación presentamos los análisis de las distintas modalidades de lectura observadas.

\subsection{Lectura compartida alternada}

Esta modalidad corresponde a aquella donde el docente y sus estudiantes comparten la responsabilidad de leer el texto, a través de los turnos de lectura asignados. De acuerdo a los análisis, esta modalidad es la más utilizada por las docentes observadas, como se mencionó previamente. A su vez, se constata que antes de iniciar la lectura, gran parte de las docentes destinan un momento para entregar instrucciones que, en su conjunto, remiten al énfasis en el tono de voz, el volumen de la voz y a respetar los signos de puntuación. Los siguientes son algunos ejemplos de esta modalidad.

Profesora: Muy concentrados en la lectura por favor, respetemos los signos, seamos expresivos para leer, ¿̇ya? Comienza, Consuelo. (Magdalena, Lenguaje $5^{\circ}$, Escuela 6, Villarrica, 2019).

Profesora: Ya, señorita Judith, comience a leer esta parte por favor, fuerte y claro. Y los demás escuchan atentos, ¿ya? (Nadia, Matemática 5, Escuela 4, Temuco, 2019).

Profesora:Todos atentos porque cuando le correspondo leer, lo va a hacer con fuerza y energía, ¿̇ya? Respetando esos signos que puedan aparecer para que nuestra lectura tenga un ritmo, ¿̇ya? (Monserrat, Lenguaje $5^{\circ}$, Escuela 7, Pucón, 2019). 
Así, constatamos que los estudiantes leyeron fragmentos cuya extensión y complejidad fue disímil, según el género discursivo y la asignatura. Utilizando la lectura compartida, se leyeron fábulas, leyendas, problemas matemáticos, instrucciones y procedimientos, fragmentos de la Biblia, poemas y algunos cuentos. Asimismo, se observó que algunas docentes, solicitaban a sus estudiantes volver a leer el mismo fragmento. Esta instrucción, tenía por finalidad aclarar algún concepto, analizar lo que se lee y enfatizar alguna información importante del texto, para así lograr una mejor comprensión. A continuación, desplegamos algunos ejemplos de la lectura compartida, de acuerdo con el texto o género discursivo que fue leído.

a) Poemas: una de las docentes, Magdalena, propone leer bajo la modalidad de lectura compartida el poema "Los ratones revoltosos". Así, constatamos su preocupación por crear un ambiente adecuado para leer, pues manifiesta que es un poema "muy divertido" y que, además, se debe leer con particular énfasis expresivo, prestando atención a las emociones comunicadas. De este modo, los estudiantes toman turnos para leer dos veces cada estrofa, intentando hacerlo de acuerdo con las indicaciones entregadas por la docente.

Profesora: Ya. Ahora vamos a la otra actividad, donde vamos a leer una poesía, ¿ya? Muy, muy divertida que se Ilama: "Los ratones revoltosos", ¿ya?, y vamos a ir tratando de decir, de leerla, pero con el corazón. La poesía se lee con el corazón, se interpreta con el alma chiquillos, ¿̇ya? Va... yo voy nombrando alguno, pero después vamos a ir turnándonos para ver ¿quién le pone más color a cada verso, ¿ya? Hay que ponerle color, icon más expresión! (Magdalena, Lenguaje, $5^{\circ}$, Escuela 6, Villarrica, 2019).

b) Problemas matemáticos: en la clase de matemáticas, las docentes observadas enseñan la resolución de problemas matemáticos. En ese contexto, emplean la lectura compartida para facilitar la comprensión del enunciado del problema. Como se advierte, resolver el problema matemático supone no solo decodificar el enunciado, sino ejercer una lectura que permita comprender su sentido. En efecto, como sucede en el caso de Nadia, se solicita que los estudiantes presten atención a la lectura del problema e, incluso, esta docente propone leer más de una vez el problema para que así sus estudiantes lo comprendan. Asimismo, se observó que otras docentes pausaban la lectura, para poder monitorear la comprensión del enunciado, como podemos observar en estos ejemplos.

Profesora: Ya, señorita Judith, comience a leer esta parte por favor, fuerte y claro. Y los demás escuchan atentos, ¿ya? ... y después me va a leer don Fernando y don Ricardo.

Estudiante: Una circunferencia es una línea curva cerrada y plana, cuyos puntos están a la misma distancia (Nadia, Matemática $7^{\circ}$, Escuela 4, Temuco, 2019).

Estudiante: (leyendo) En el centro médico centenario se atienden distintas especialidades. El traumatólogo demora un cuarto de hora en atender a cada paciente. El estuvo de corrido examinando a sus pacientes. ¿Cuantas horas horas estuvo trabajando el doctor de Traumatología? (Pamela, Matemática 6², Escuela 2, Villarrica, 2019).

Profesora: Les voy a hacer leer tres veces el problema, porque quiero que vayan ustedes también pensando. Sabina, léame el problema... y después me va a leer José y el Sr. Urrutia, iYa! ¿qué dice?

Estudiante: Juan construyó un corral rectángulo de 21 metros de largo, cuyo perímetro es de 78 metros ¿Cuál es el ancho del corral de Juan? (Nadia, Matemática 5², Escuela 4, Temuco, 2019).

c) Ensayos de ética y la Biblia: En la clase de Religión, pudimos observar que la lectura compartida se utilizó para leer fragmentos de la Biblia y ensayos filosóficos. A diferencia de lo observado en otros casos de esta modalidad, son los alumnos quienes llevan a cabo la lectura de manera continuada. Además, constatamos que la docente no destina ni un momento para explicar cómo leer adecuadamente el texto en voz alta. En general, sus intervenciones solo se limitaron a esclarecer algún concepto puntual del fragmento leído.

Estudiante 1: "Las reflexiones teológicas o filosóficas sobre la situación de la humanidad y del mundo pueden sonar repetidas y abstractas, si no se presentan nuevamente a partir de una confrontación con el contexto actual, en lo 
que tiene de inédito para la historia de la humanidad. Por eso, antes de reconocer cómo la fe aporta nuevas motivaciones y exigencias frente al mundo del cual formamos parte, propongo de te, de te, dete-nernos a considerar lo que le está pasando a nuestra casa común". Ya, amiga, tú tienes que leer el 18 y el 19.

Estudiante 3: "A esto se suma el problema de que los objetos, que los objetivos de ese cambio veloz y constante no nece....no necesariamente se orientan al bien común y a un desarrollo humano, sostenible e inten...integral. El cambio es algo deseable, pero se vuelve preocupante cuando se convierte en deterior, deterioro... del mundo y de la calidad de vida de gran parte de la humanidad" (Nicol, Religión, 6º Escuela 3, Temuco, 2019).

d) Textos instructivos: En esta clase de textos, pudimos observar que la lectura compartida se lleva a cabo mediante la estrategia de segmentar las instrucciones del texto para asignar los turnos de lectura. En efecto, como sucede en el caso de Elisa, para favorecer la comprensión ella aclara que "vamos a rescatar algunas cosas que son significativas de este texto". No obstante, a diferencia de lo que sucede con otros casos de lectura compartida, en esta oportunidad no se destaca la importancia de elementos como el tono de voz, la pronunciación o que se respeten los signos de puntuación, como podemos ver en el siguiente ejemplo.

Profesora: Puedes guiar un poquito la lectura de Ana, por favor. Ya, Maira, me harías el favor de leer esta parte, que inicia el texto que acabamos de trabajar, desde los tres primeros tú.

Estudiante 1: Materiales: molde de máscara, hoja de cartulina, colores.

Estudiante2: Tijeras, cinta elástica.

Estudiante 3: El pegamento líquido, pedazos de papel de colores.

Profesora: Bien, Antonia... Le pediría que se siente bien, sentadita. Entonces, chicos, voy a leer solamente el título, la primera instrucción, ¿̇ya? Ustedes van a leer sólitos la primera, nos vamos a ir dividiendo de uno a uno y vamos a rescatar algunas cosas que son significativas de este texto.

Estudiante 4: "Instrucciones: Dibuja en la cartulina el contorno de la máscara. Traza el contorno de los ojos y los agujeros en cada extremo para ajustar... la cinta".

Profesora: Bien. "Recorta el contorno de la máscara y el de los ojos".

Estudiante 5: “Colorea la máscara con ayuda de crayones” (Elisa, Lenguaje 3², Escuela 7, Pucón, 2019).

e) Textos narrativos: En este caso, constatamos que la lectura compartida se llevó a cabo a través de diversos géneros narrativos como el cuento, la fábula y la leyenda. A su vez, observamos que los estudiantes mostraban una mejor disposición para colaborar con la lectura. Probablemente, esto se vincula con las mayores posibilidades expresivas y motivacionales que caracteriza a los textos literarios. No obstante, las docentes también contribuyeron a crear esta disposición favorable en los estudiantes. Por ejemplo, Deborah refuerza positivamente al estudiante que asume el turno para leer y Monserrat hace lo propio sugiriendo que se lea "con energía".

Profesora: Muy bien. "Era un enorme sapo, muy divertido por los saltitos de la pulga, que parecían imitar sus enormes saltos. Le preguntó que a dónde iba. -Voy a los juegos -repuso la pulga- a llamar al nieto de una pobre anciana solitaria que lo espera en su choza".

Estudiante 1: "-Voy a los juegos -repuso la pulga- a llamar al nieto de una pobre anciana solitaria que lo espera en su choza. Sí es así -dijo el sapo- yo te, yo te llevaré en mi boca y llegarás mucho más rápido".

Profesora: Sí, muy bien. "Aceptó agradecida la pulga, instalándose en la bocaza del sapo".

Estudiante 2: “No había avanzado mucho el sapo saltón con su pulga saltarinas cuando los alcanzó, los alcanzo una larga culebra. Y, como hacen todos los viajeros que alcanzan a otro en el camino, quiso iniciar una conversación con el sapo: -¿No te duelen las patas de tanto saltar y saltar?". 
Profesora: Muy bien. "Algo maltratado, - dijo el sapo -, pero llevo en mi boca una pulga que va a los juegos de pelota a buscar al nieto de una anciana solitaria, que lo espera en su choza. En ese caso, dijo la culebra, - yo te tragaré y todos llegaremos más rápido".

Estudiante 3: "-¿iNo te duele el vientre arrastrándote tan rápido por piedras y espinas? - preguntó el ave".

Profesora: Muy bien. "- algo me duele-, respondió la culebra, pero llevo en mi estómago un sapo, que lleva en su boca una pulga, que va a los juegos de pelotas a buscar al nieto de una anciana solitaria que lo espera en su choza".

Estudiante 4: "En ese caso, dijo el aguilucho, -yo te alzaré en mis garras y todos llegaremos más rápido. Aceptó agradecida la culebra y así continuó con vía aérea" (Deborah, Lenguaje $5^{\circ}$, Escuela 5, Temuco, 2019).

Profesora: Empezamos, dice así: "Las Tres Marías. Don Ventura es una persona distinguida en Doñihue, donde se encuentra arraigado desde su tierna y juguetona infancia, se estima especialmente tanto por sus bellas condiciones, como por la virtud de su dinero. Es dueño de un gran establecimiento comercial. Que comprende tiendas de trapo, mercería, abarrotes y hasta un esbozo de botica..." Siga...

Estudiante 1: "Ahí, ahí me detuve una mañana a contemplar las 3 Marías... vista recreativa del cerro... hacia el Norte...cielo...".

Estudiante 2: "Llegaron luego a participar luego del entusiasmo ...el alcalde de la comuna... hablamos de cuestiones temas nuevos. Pregúntele usted al mío, me dijo el alcalde guiñándome un ojo porque le había puesto a su establecimiento Las tres Marías".

Profesora: Hasta ahí. Muchas gracias... usted con energía por favor. Mi novel.

Estudiante 3: "Tenía yo 26 años y una chalita, por esto... Una salud excelente según me lo declararon alguna vez cuando yo era joven y simpático. Entonces, llegó aquí a radicarse la señora Ignacia. Una hermosa mujer llena de esa belleza bizarría...".

Profesora: Muchas gracias. Siga usted (Monserrat, Lenguaje 5², Escuela 7, Pucón, 2019).

Como podemos constatar, al ser más frecuente, esta modalidad se utilizó para mediar diversos tipos de textos, en distintas asignaturas, lo que sugiere que la instancia de leer en conjunto, no necesariamente se utiliza solo con los textos literarios. Por otro lado, dado que la evidencia sugiere que un clima afectivo-emocional cálido durante la lectura incrementa en los estudiantes la atención al texto, la cooperación con el docente y el entusiasmo por participar de la lectura (Landry et al., 2012); en las clases analizadas -especialmente en aquellas donde se comparte la lectura de textos narrativos- se observó una mayor disposición de los estudiantes por participar en esta instancia. Asimismo, gran parte de las docentes estudiadas que emplearon esta modalidad, se preocuparon por destacar aspectos vinculados con el tono de voz, la pronunciación, el volumen de la voz, y la capacidad expresiva. Sin embargo, de acuerdo con nuestros análisis, la mayor parte de ellas no destina un tiempo para modelar frente a sus estudiantes la puesta en práctica de estos aspectos.

\subsection{Lectura compartida grupal y al unísono}

En esta modalidad de lectura compartida, el docente solicita a sus estudiantes que lean en voz alta al unísono de forma conjunta el texto que es abordado en la clase. De acuerdo con las evidencias, esta modalidad también es ocupada con cierta frecuencia por las docentes observadas. A su vez, también constatamos la tendencia de utilizar diversos tipos de textos como poemas, fábulas y el texto instructivo. Para que la lectura se llevara a cabo sin problemas, las docentes propiciaron un ambiente favorable, solicitando a sus estudiantes que leyeran tranquilamente, sin apurarse, y precupándose por los estudiantes que presentaban menos fluidez en la lectura.

Por último, los cursos donde se llevó a cabo este tipo de lectura más coral, estaban integrados por estudiantes de ambos géneros, no obstante, en una de las escuelas observadas, la docente solicitó a los estudiantes que 
pudieran leer fragmentos de un poema de forma conjunta, pero organizando los turnos entre hombres y mujeres. Algunos de los fragmentos que ejemplifican la lectura grupal en voz alta son:

Profesora: Ahora la vamos a leer de otra manera, ¿̇ya? Están... siempre les he dicho tanto niños como niñas tienen la misma, pueden hacerlo igual de bien, ¿no es cierto? Y ahora lo vamos a demostrar. Primera estrofa empeza... La tía va a decir el titulo y el autor. Comienzan los varones con la primera estrofa y así nos vamos alternando, ¿ya? Niñas, varones, niñas. Muy atentos, la voz tiene que salir más fuerte. Que resalte el sentimiento. ¿Estamos? Ya. Nos concentramos entonces, vamos a declamar, vamos a poner el sentimiento. (Texto: "Los ratones revoltosos") (Magdalena, Lenguaje 5, Escuela 6, Villarrica, 2019).

Profesora: Como voy a ir leyendo arriba, como lo hacemos en forma habitual (Alan), lo hacemos en conjunto sin ir apurando, yo sé que aquí hay niños que leen súper rápido, pero ya lo vamos hacer de manera compartida.

Todos: "Materiales, molde de máscara, hoja de cartulina, crayones o tempera, tijeras, cita elástica, pegamento líquido, retazos de papel de colores" (Elisa, Lenguaje 3², Escuela 7, Pucón, 2019).

Profesora: Leamos todos hasta el punto que viene. Solo hasta el punto (todos juntos).

Todos: "Pocos días después unos cazadores apresaron al rey de la selva y le ataron con una cuerda a un frondoso árbol".

Profesora: ¿Y si leemos todos la moraleja?

Todos: Sí!!! "Nunca desprecia las promesas de los pequeños honestos cuando llegue el momento las cumplirán" (Elisa, Lenguaje $3^{\circ}$, Escuela 7, Pucón, 2019).

\subsection{Lectura en voz alta del profesor}

A diferencia de la lectura compartida, en esta modalidad, el profesor asume un mayor protagonismo, pues él es quien lee en voz alta para sus alumnos. Por tanto, la forma en cómo se presentan dimensiones relacionadas con el tono de voz, la pronunciación, la entonación y los énfasis expresivos cobran particular interés, sobre todo considerando que después de la lectura compartida, esta modalidad fue la más utilizada.

Al momento de leer para sus alumnos, algunas docentes primero resaltan la importancia de manejar una entonación adecuada, de acuerdo con los signos de puntuación, así como respetar las pausas, silencios, y el tono interrogativo o exclamativo de las preguntas. Así, enfatizar estos aspectos, aparece asociado a lograr una mejor comprensión del texto leído. Por ello, para el propósito de captar la atención de los estudiantes y así desarrollar una lectura expresiva, atractiva y comprensible para ellos, la mayor parte de las docentes se preocupa por emplear un tono adecuado, así como una entonación que resalta algunas palabras o frases claves del texto. A continuación, presentamos algunas evidencias.

Profesora: Ya... ahora ustedes me van a escuchar a mí, como yo les voy a leer, porque les voy a modelar la lectura. Ya, atención, atención, yo les voy a modelar la lectura, dice: "Juan construyó un corral rectángulo de 21 metros de largo, cuyo perímetro es de 78 metros ¿Cual es el ancho del corral de Juan?". ¿Se escucha diferente? (Nadia, Matemática $5^{\circ}$, Escuela 4, Temuco, 2019).

Profesora: Bien. Voy a leer la tres. "Recorta el contorno de la máscara y el de los ojos? Bien, yo voy a repasar nuevamente las instrucciones. Le voy a poner énfasis en las palabras que estamos buscando, ¿ya? Dibuja en la cartulina contorno de la máscara. Traza el contorno de los ojos y los agujeros uno en cada extremo para anudar las cintas. Recorta el contorno de la máscara y el de los ojos. Colorea la máscara con ayuda de tus crayones, si utilizas témpera deberás esperar a que se seque antes de seguir trabajando, de lo contrario puedes mancharte. Agrega un poco de pegamento en el contorno de tu máscara y pega los trozos de papel de colores. Anuda la cita elástica en los agujeros que hiciste los extremos de tu máscara. Sujeta la máscara tu cabeza y.." (Elisa, Lenguaje $3^{\circ}$, Escuela 7 , Pucón, 2019). 
Profesora: No. Atención, dice así, texto número dos. Dice así... Ya, dice así. Shhhh, dice: Matilda Ronald Dahl, escritor Británico. "Antes de que terminara la primera semana del curso, ya circulaban entre los alumnos impresionantes historias sobre la directora, la señorita Trunchbull. -Basura nueva, supongo, dijo Hortensia, mirándola despectivamente. Llevaba una bolsa gigante de patatas fritas, que comía a puñados. -Os ha tocado un premio, dijo Hortensia. Odia a las niñas muy pequeñas. Por eso aborrece el curso infantil y todo lo que se relaciona con él. Cree que los niños de cinco años son gusanos que aún son larvas. -¿Qué está haciendo aquí está niñita en algún momento?" (Deborah, Lenguaje 6º Escuela 5, Temuco, 2019).

Profesora: "Visitó muchos planetas y al segundo planeta que llegó, estaba a habitado por un vanidoso. Ahhh un admirador viene a visitarme, grito el vanidoso al divisar a lo lejos al Principito. Para los vanidosos todos los demás hombres son admiradores. -iBuenos días! -dijo el Principito-. iQué sombrero tan raro tiene! - Es para saludar a los que me aclaman -respondió el vanidoso. Desgraciadamente nunca pasa nadie por aquí. -¿Ah, sí? -preguntó sin comprender el Principito. -Golpea tus manos una contra otra, le aconsejó el vanidoso. El Principito aplaudió y el vanidoso le saludó modestamente levantando el sombrero" (Mariela, Lenguaje 4", Escuela 7, Pucón, 2019).

A partir de las evidencias, el respectivo análisis apunta hacia dos consideraciones generales. En primer lugar, como sucedió con la lectura compartida, observamos una tendencia similar en términos de que se utilizaron diversos tipos de textos; por ende, la lectura del profesor fue una modalidad que se puso en práctica en varias asignaturas y no solo en Lenguaje y Comunicación. Este último punto es importante, dado que concuerda, por ejemplo, con el planteamiento que al respecto se formula en el libro "A viva Voz. Lectura en Voz alta" (Ministerio de Educación, 2013), donde se destaca que este tipo de lectura es importante para todas las disciplinas.

En segundo lugar, constatamos que las docentes observadas se preocupan por llevar a cabo una lectura en voz alta que sea fluida, donde además se resaltan algunas palabras o frases del texto. Sin embargo, profundizando un poco más, cabría destacar que en estas docentes se presentan estilos distintos, sobre todo en el momento de iniciar la lectura. En específico, mientras algunas prefieren explicar a sus estudiantes algunos aspectos para desarrollar una adecuada lectura en voz alta, como sucede con Nadia, otras optan por iniciar la lectura sin explicaciones previas, como en el caso de Deborah. De este modo, explicar a sus estudiantes algunas dimensiones importantes de la lectura en voz alta, o bien, resaltar alguno de sus beneficios, es característicos solo de alunos casos observados. En esa línea, advertir este último aspecto es importante porque la lectura del profesor es considerada como uno de los factores influyentes en el desarrollo integral de los estudiantes (Chambers, 2001; Trostle \& Donato, 2001; Van den Broek et al., 2005), por sus beneficios asociados a aumentar el vocabulario en contexto, reconstruir y ampliar las estructuras mentales de los estudiantes, así como promover la conciencia fónica, facilitando la comprensión y el desarrollo de estructuras segmentales y prosódicas de la lengua (Trostle \& Donato, 2001).

\subsection{Lectura silenciosa}

Como su nombre lo indica, se refiere al tipo de lectura de un mismo texto o de textos distintos por parte de los estudiantes, de forma silenciosa e individual, en donde la concentración y el clima de la clase juegan un rol muy importante al momento de comprenderlo. Esta modalidad, tradicionalmente conocida como lectura silenciosa sostenida, cuenta con una larga tradición en el sistema escolar chileno (Condemarín, 1987). Como este tipo de lectura es uno de los menos frecuentes en las escuelas observadas, solo una docente la implementó con sus estudiantes en dos momentos distintos de la clase. Así, la profesora explicitó a sus estudiantes que leerían el mismo fragmento de un texto de forma silenciosa, para lo cual preparó un clima favorable en el aula para posteriormente pasar a una actividad donde los estudiantes comparten y comentan lo leído.

Profesora: Ya, chicos entonces el tema de trabajo de hoy día, vamos a reflexionar ya, sobre la importancia que tienen las plantas y el medio ambiente que nos rodea, ¿̇ya? Y nuestra tarea es leer un texto, primero lo van a leer en silencio, luego lo vamos a proyectar acá adelante y lo vamos a analizar y a conversar. Para posteriormente poder responder, unas preguntas que nos van a servir de análisis, y para que podamos sacar algunas, ideas claras del tema del medio 
ambiente y las plantas. Por favor tomen su hojita en forma silenciosa, cada uno... vamos a iniciar esta vez la lectura silenciosa y luego una lectura compartida, ¿̇ya? (Elisa, Ciencias Naturales $3^{\circ}$, Escuela 7, Pucón, 2019).

Profesora: Muy bien, voy a pedir que ahora bajemos el volumen un poquito. Y Cada uno silencio, en silencio, en silencio va a leer la primera parte de su texto que tienen ahí, y luego yo le voy a pedir alguien que me ayude con la lectura acá. ¡Ya! bajamos la voz para permitir que los compañeros que tienen dificultad de lectura, pueden leer tranquilitos ¿Partimos? (Elisa, Lenguaje $3^{\circ}$, Escuela 7, Pucón, 2019).

\subsection{Lectura Independiente}

Una de las principales características de esta modalidad es que el docente propicia un tiempo para que sus estudiantes puedan escoger libremente algún texto para leer. Por ello, este tipo de lectura se asocia al propósito de favorecer la lectura por placer e, incluso, constituye una estrategia pertinente para incrementar el uso sistemático de la bibioteca escolar y formar lectores activos (Lockwood, 2011).

El análisis muestra que la lectura independiente es una de las modalidades menos utilizada por las docentes observadas. En ese sentido, las oportunidades de aprendizaje que se ofrecen para promover la lectura por placer, serían más bien limitadas y, como se observó, se presentan solo en la asignatura de Lenguaje y Comunicación. No obstante aquello, cabe señalar que una de las docentes, Deborah, desarrolla esta instancia en la biblioteca de su escuela, por lo tanto, sus estudiantes tienen la posibilidad de familiarizarse con la colección bibliográfica que pone a disposición este espacio. Esto es particularmente relevante dado que, en general, existe un limitado uso de las bibliotecas escolares y casi no se consideran las preferencias lectoras de los estudiantes (Gelber, 2017).

Por último, el poner en práctica este tipo de lectura, como sucede con Deborah y Miriam, presupone desarrollar una actividad posterior; es decir, luego de la lectura se propicia un momento para compartir y comentar lo que cada estudiante leyó. En efecto, esta instancia es también una oportunidad para la discusión y la reflexión no solo sobre el texto o las interpretaciones del estudiante, sino que además acerca del proceso metacognitivo de cada lector. De este modo, a pesar de su carácter individual, la actividad tiene finalmente una proyección más dialógica. A continuación, desplegamos algunos ejemplos.

Profesora: ¡Ya! A ver... ¿todos están con su libro? Rapidito, asiento, asiento. Shhh (en biblioteca).

Estudiante 1: Tengo unas consultas, ¿Qué hay que hacer?

Profesora: ¡Ya! ... van a tener unos minutos para leerlo y después van a ... para saber si comprendieron o no. Y eso es a partir de...

Estudiante 2: ¿Y hay que inventar las preguntas?

Profesora: Chicos, en cinco minutos voy hacer que algunos alumnos pasen a delante, a contar de que se trató su texto y qué hicieron para poder comprenderlo, que estrategia utilizaron. (Deborah, Lenguaje $6^{\circ}$, Escuela 5, Temuco, 2019)

Profesora: Si, por favor. Bien, Échenle un vistazo para luego decirles lo que vamos a hacer con el libro, pero conózcalo primero por favor (Miriam, Lenguaje $7^{\circ}$, Escuela 1, Villarrica, 2019).

\subsection{Lectura dramatizada}

Esta modalidad de lectura tiene como finalidad que los estudiantes del curso puedan actuar con la voz lo que están leyendo, es decir, tienen que realizar la lectura como si la estuvieran viviendo. Así, coloca el énfasis en la expresividad, el ritmo, el tono y volumen de la voz, entonación y en la mejora continua de la fluidez en los 
estudiantes. Cabe destacar que esta modalidad, al ser más activa, lúdica y teatral, en general, produce mayor motivación en los estudiantes (Gelber, 2017).

De acuerdo con los análisis, esta modalidad es una de las que reporta la menor cantidad de episodios, como señalamos anteriormente, pues solo dos de las docentes la utilizaron en su clase de Lenguaje. De igual modo, tanto Magdalena como Mariela propusieron textos narrativos como el cuento "El león enamorado" y un fragmento del "Principito" respectivamente, esto sugiere que los textos literarios, serían los más pertinentes para poner en práctica la lectura dramatizada. En efecto, parece ser que esta modalidad, por su función teatral, está más vinculada al área de lenguaje, pese a que podría ser un recurso útil y motivador en otras asignaturas. Por útlimo, siguiendo con el caso de Magdalena, también se observa una preocupación por preparar la lectura, en términos de asignar los personajes a los estudiantes que van a leer, enfatizar que se debe leer con expresión, y finalmente, explicar la actividad a desarrollar una vez que termine la lectura, sin desatender el contenido del texto. Así también lo demuestra Mariela, quien destaca que se siga con atención la lectura, porque algunos estudiantes asumirán el rol de un personaje de la historia, como podemos comprobar a continuación.

Profesora: "Todos tienen que ver claro, acomódense mirando hacia acá, que puedan ver... ya. Vamos a dividirnos así presten mucha atención (siéntate bien por favor), eh...desde Consuelo hacia acá va a ser León, ¿̇ya? por favor, desde Consuelo hacia acá. Desde Bastián hasta Amanda, van hacer hija y desde ustedes dos hacia acá, van hacer el labrador. ¿ya? Leamos con expresión, en el teatro uno no habla parejito, ¿̇no es cierto?, eso lo hemos aprendido. Tiene que ser con expresión..., ya...se llama el León enamorado. Ya chiquillos, la vamos a leer una vez más, muy rápido. Pero vamos a repartir el rol. ¿Quién va ha ser el león?, Amanda va a ser la hija. ¿Cuál es la Amanda?, y Sebastián Cisternas va a ser el labrador. Pongan mucha atención en el contenido, también en las acotaciones que hay, porque después vamos a llenar una ficha relacionada con lo que van a leer" (Magdalena, Lenguaje $5^{\circ}$, Escuela 6 , Villarrica, 2019).

Profesora: Ahí es donde tenemos que estar atentas, porque ustedes van a ser el otro personaje. Tienen que estar siguiendo la lectura a los que les va a tocar hablar, leer, ¿̇ya?. Vamos... ya, empieza tú, tú eres el zorro. (Lectura del texto "El Principito")

Estudiante 1: "Buenos días".

Profesora: "Buenos días"

Estudiante 2: "Estoy aquí abajo bajo un Manzano".

Profesora: “¿Quién eres?, Eres muy bonito".

Estudiante 3: "Soy un zorro, no puedo domesticarlo".

Profesora: "Ah... perdón no estoy domesticado. ¿Qué significa domesticar?".

Estudiante 4: "Tú no eres de aqui"

Profesora: "Busco a los hombres, ¿̇qué significa domesticar?"

Estudiante: "Los hombres... cazan muy molestos, aunque también las gallinas, ¿̇uscas gallinas?” (Mariela, Lenguaje $4^{\circ}$, Escuela 7, Pucón, 2019).

\section{Conclusiones}

Entonces, ¿qué podemos aprender de las modalidades de lectura en las prácticas pedagógicas de las profesoras participantes? Por ejemplo, si consideramos los resultados del estudio de Gelber (2017), que abarcó una muestra de alumnos y profesores desde primero a cuarto medio, la lectura en voz alta del docente y los estudiantes y la silenciosa destacan como las modalidades que más utilizan los profesores. En efecto, por un lado, nuestros 
resultados muestran que la lectura compartida y la lectura del profesor, ambas modalidades en voz alta, son las más frecuentes. Por ende, la experiencia de leer junto a otros con la participación activa del docente, como lo propone la lectura compartida, así como la lectura del profesor, que permite poner en escena un modelo de lectura en voz alta, serían instancias de mediación lectora que parecen presentar una trayectoria continua en los niveles educativos del sistema escolar. Por otro lado, respecto de los resultados de Gelber (2017) sobre la lectura silenciosa, las prácticas de las docentes observadas en este estudio presentaron un menor uso de esta modalidad. Entonces, sería necesario indagar si efectivamente la lectura silenciosa se utiliza menos en la educación básica, a través de la observación directa de las prácticas pedagógicas, especialmente considerando que el estudio de Gelber (2017), relevó las actividades de lectura solo a través de encuestas y entrevistas.

Asimismo, el citado estudio también detectó que la lectura dramatizada y la lectura independiente, son las modalidades menos utilizadas por los profesores. En consecuencia, como nuestros resultados siguen esa tendencia, cabría consignar que las oportunidades de aprendizaje vinculadas a que los estudiantes pongan en práctica los recursos expresivos que presupone la lectura dramatizada, se presentan de forma limitada a nivel transversal. En efecto, dado que mediante este tipo de modalidad "se ofrece un modelo de lectura expresiva, entusiasta; se transmite el gusto por leer, y se invita a los auditores a ser lectores" (Richardson, 2000, p. 3), aparece como un factor importante, profundizar con otros estudios las razones que explicarían la baja utilización de la lectura dramatizada en las prácticas docentes de enseñanza básica. De igual modo, por la importancia que nuestro currículo le asigna a la formación de lectores, en términos de que los estudiantes vayan progresivamente afianzando sus hábitos lectores así como sus preferencias, es preocupante que nuestros resultados detecten una baja presencia de la lectura independiente, como también lo hace Gelber (2017). Probablemente, este resultado se vincula, por ejemplo, con las evidencias sobre la disminución de las actitudes lectoras positivas del alumnado a medida que pasa de un curso a otro (Errázuriz et al., 2020; Gelber, 2017).

Los resultados presentandos nos permiten afirmar que todas las docentes observadas, al momento de mediar un texto, ponen en práctica alguna de las modalidades de la lectura en voz alta. Sin embargo, cabría advertir que esto no necesariamente significa que se hayan utilizado del mismo modo. Más bien, nuestros resultados sugieren que existen estilos docentes particulares, incluso bajo la misma modalidad. Por ejemplo, mientras algunas docentes destinan un momento para formular sugerencias a sus estudiantes asociadas a usar un tono de voz adecuado, respetar los signos de puntuación e incluso manejar un volumen adecuado, en otros casos, no se observa esta situación. Otra profesora, Mariela, incluso utilizó varias modalidades de lectura para leer un mismo texto, motivando así a los estudiantes de modo activo. Asimismo, un aspecto que sí se presentó de forma transversal en las prácticas de estas docentes, es que al momento de leer en voz alta, no necesariamente se explica a los estudiantes los beneficios de este tipo de lectura.

Asimismo, como estrategias que colaboran con la mediación lectora, las distintas modalidades de lectura en voz alta obervadas, son bastante útiles para abordar los diversos tipos de textos que cada asignatura propone de acuerdo a su itinerario curricular. En efecto, aun cuando la mayor diversidad estuvo asociada a la literatura, de igual forma se constató que tanto la Biblia como los textos instructivos y los problemas matemáticos, pueden ser objeto de una mediación a través de la lectura en voz alta. Estos casos, sirven de ejemplo para poner en relieve, los planteamientos que advierten la importancia de que la lectura en voz alta sea una estrategia presente en todas las asignaturas, y no solo en Lenguaje y Comunicación (Ministerio de Educación, 2013). En ese sentido, sí nos llama la atención la escasa presencia de géneros discursivos expositivos o informativos, pues solo se presentaron textos instructivos, especialmente considerando que los participantes fueron profesoras de distintas asignaturas y que estos textos son frecuentes en los ámbitos de las iencias y las ciencias sociales. Por tanto, podemos constatar que las evidencias mencionadas sobre los bajos índices de lectura de textos expositivos en educación básica, debido a la preeminencia de géneros narrativos con los cuales los estudiantes están más familiarizados, se aplican en este caso (De Mier, Amado y Benítez, 2015; Errázuriz et al, 2020; Ruiz, Montenegro, 
Meneses y Venegas, 2016). Esta falta de diversidad de géneros textuales, ofrecería menores posibilidades de presentar a los alumnos textos más elaborados que pueden contribuir al desarrollo de un diálogo más sofisticado (Anderson, Lynch, Shapiro \& Eun Kim, 2012).

Por ultimo, respecto de las proyecciones de esta investigación, consideramos que es necesario continuar investigando cómo se presentan las modalidades de lectura en las prácticas pedagógicas, pero incorporando entrevistas a los docentes, así como también ampliar el número de casos a observar. De este modo, podría profundizarse aun más en distinguir con mayor claridad el tipo de contribución que brinda la lectura en voz alta, pero en el contexto de la mediación de la lectura en las asignaturas. En este sentido, es necesario afianzar líneas de investigación que consideren un amplio conjunto de resultados sobre la puesta en práctica de las modalidades de lectura en voz alta, por parte de los docentes, pero en función de analizar las posibles brechas existentes con las conceptualizaciones y lineamientos pedagógicos que se formulan a propósito de la lectura en voz alta, tanto en los documentos curriculares como en los que apoyan su implementación.

\section{Referencias bibliográficas}

Alexander, R. J. (2008). Culture, dialogue and learning: Notes on an emergent pedagogy. In N. Mercer \& S. Hodgkinson (Eds.), Exploring talk in school (pp. 93-114). London, Reino Unido: Sage.

Anderson, A., Anderson, J., Lynch, J., Shapiro, J. \& Eun Kim, J. (2012). Extra-textual talk in shared book reading: A focus on questioning. Early Child Development and Care, 182(9), 1139-1154. DOI: 10.1080/03004430.2011.602189

Barber, M. y Mourshed, M. (2008). Cómo hicieron los sistemas educativos con mejor desempeño del mundo para alcanzar sus objetivos. Santiago: CINDE McKinsey \& Company.

Bazerman, C., Dean, C., Early, J., Lunsford, K., Null, S., Rogers, P. \& Stansell, A. (eds.). (2012). International advances in writing research: Cultures, places, measures, Perspectives on writing. Fort Collins, CO: The WAC Clearinghouse and Parlor Press.

Becerra, R. y Mayo, J. S. (2015). Percepciones acerca del rol de las comunidades mapuche en un jardín intercultural bilingüe. Psicoperspectivas, 14(3), 56-67.

Benevides, T. \& Peterson, S. (2010). Literacy attitudes, habits and achievements of future teachers. Journal of Education for Teaching, 36(3), 291-302.

Bustos-Ibarra, A. V., Montenegro, C. S., Jarpa-Azagra, M. V., Calfual-Catalán, K. P. y Tapia-Ibacache, A. (2019). En las salas de primaria ¿Cómo se lee para aprender?. Revista ESPACIOS, 40(41), 25.

Carlino, P. (2005). Escribir, leer y aprender en la universidad. Una introducción a la alfabetización académica. México: Fondo de Cultura Económica.

Cassany, D. (2006). Tras las líneas: Sobre la lectura contemporánea. Barcelona: Graó.

Chambers, A. (2001). El lector en el libro. En M. F. Bellorín y P. Castillo, Un encuentro con la crítica y los libros para niños. Caracas: Banco del Libro.

Chartier, A. y Hébrard. (2000). Saber leer y escribir: unas 'herramientas mentales' que tienen su historia. Infancia y Aprendizaje, 23(89), 11-24.

Condemarín, M. (1987). El programa de lectura silenciosa sostenida. Santiago: Andrés Bello. 
Consejo Nacional de la Cultura y las Artes. (2015). Política Nacional de la Lectura y el Libro 2015-2020. Santiago: CNCA.

Cunningham, Anne E. \& Keith E. Stanovich (1998). What reading does for the mind. Journal of Direct Instruction, 1 (22),137-149.

De Mier, M. V., Amado, B. y Benítez, M. E. (2015). Dificultades en la comprensión de textos expositivos en niños de los primeros grados de la escuela primaria. Psykhe, 24,(2), 1-13.

Dickinson, D., Golinkoff, R. \& Hirsh-Pasek, K. (2010). Speaking out for language: Why language is central to reading development. Educational Researcher, 39(4), 305-310. Recuperado de: https://www.jstor.org/ stable/27764601

Errázuriz, M. C. (2017). Teorías implícitas sobre escritura académica de estudiantes de programas de formación inicial docente: ¿Inciden en el desempeño escrito?. Signo y Pensamiento, 36(71), 34-50.

Errázuriz, M. C. (2019). Desempeño escrito de estudiantes de programas de formación inicial docente: ¿Cómo es la calidad del proceso de escritura de sus ensayos?. Lengua y Habla, 23, 224 - 242. Recuperado de: http://erevistas.saber.ula.ve/index.php/lenguayhabla/article/viewFile/15670/21921926771

Errázuriz, M. C., Becerra, R., Cocio, A., Davison, O. y Fuentes, L. (2018). Concepciones sobre lectura de profesores en ejercicio: Perfiles lectores de docentes y su relación con el desempeño de sus estudiantes. Informe final FONIDE $N^{\circ}$ FX11619. Santiago: Mineduc.

Errázuriz, M. C., Becerra, R. Aguilar, P. Cocio, A., Davison, O. y Fuentes, L. (2019). Perfiles lectores de profesores de escuelas públicas de la Araucanía, Chile: Una construcción de sus concepciones sobre la lectura. Perfiles Educativos, XLI(164), 28-46. Recuperado de: http://www.scielo.org.mx/pdf/peredu/v41n164/0185-2698-peredu-41-164-28.pdf

Errázuriz, M. C., Fuentes, L., Cocio, A., Davison, O., Becerra, R. y Aguilar, P. (2020). ¿Comprendemos más los textos si nos gusta leer?: Actitudes y desempeños lectores del estudiantado de escuelas públicas de la Araucanía, Chile. Revista Brasileira de Linguística Aplicada, 20(3) (en prensa).

Flyvbjerg, B. (2006). Five misunderstandings about case-study research. Qualitative inquiry, 12(2), 219-245.

Freire, P. (2012). Pedagogía del oprimido. Buenos Aires: Siglo XXI.

Gelber, D. (2017). Documento de Trabajo №6. Mis Lecturas Diarias y valoración de la lectura en enseñanza media. Santiago: Mineduc.

Hargrave, A. C. \& Sénechal, M. (2006). A book reading intervention with preschool children who have limited vocabularies: The benefits of regular reading and dialogic reading. Early Childhood Research Quarterly, 15(1), 75-90.

Hoffman,J.L.(2011).Co-constructing meaning: Interactive literary discussions in kindergarten read-alouds. The ReadingTeacher 65(3),183-94. DOI:10.1002/TRTR.01025

Iturra, C. (2014). Los diálogos construidos y los contenidos elaborados en clases dedicadas a la comprensión de textos, en aulas chilenas. Educational Psychology, 19(2), 113-122.

Kucan, L. \& Palincsar, A. (2013). Comprehension instruction through text-based discussion. Newark, DE: International Reading Association. 
Landry, S. H., Smith, K. E., Swank, P. R., Zucker, T., Crawford, A. D. \& Solari, E. F. (2012). The effects of a responsive parenting intervention on parentchild interactions during shared book reading. Developmental Psychology, 48, 969-986. DOI:10.1037/a0026400

Larraín, A., Howe, C., y Cerda, J. (2014). Argumentación en Enseñanza en Clase Completa y Aprendizaje de Ciencias. Psykhe, 23(2), 1-15.

Larraín, A., Strasser, K. y Lissi, M. R (2012). Lectura compartida de cuentos y aprendizaje de vocabulario en edad preescolar: un estudio de eficacia. Estudios de Psicología, 33(3), 379-383. DOI: $10.1174 / 021093912803758165$

Latorre, M. (2014). Estudio Mis lecturas diarias y valoración de la lectura $5^{\circ}$ a $8^{\circ}$ básico. Santiago: Mineduc.

Lever, R. \& Sénéchal, M. (2011). Discussing stories: On how a dialogic reading intervention improves kindergartners' oral narrative construction. Journal of Experimental Child Psychology, 108(1), 1-24.

Liston, D., Borko, H. \& Withcomb, J. (2008). The teacher educator's role in enhancing teacher quality. Journal of Teacher Education, 59(2), 111-116.

Lockwood, M. (2011). Promover el placer de leer en la educación primaria. España: Morata.

Llopart, M. \& Esteban-Guitart, M. (2018). Funds of knowledge in 21st century societies: inclusive educational practices for under-represented students. A literature review. Journal of Curriculum Studies, 50(2), 145161, DOI: 10.1080/00220272.2016.1247913

Manzi, J. y García, M. R. (Ed.) (2016). Abriendo as puertas del aula. Transformación de las prácticas docentes. Santiago: Ediciones UC.

McGee, L. \& Schickedanz, J. (2007). Repetead interactive read-alouds in preschool and kindergarten. The Reading Teacher, 60(8), 742-751. DOI: 10.1598/RT.60.8.4

Meneses, A., Müller, M., Hugo, E. y García, Á. (2016). Discusión productiva para la comprensión de textos: habilidades y conocimientos específicos en la formación inicial de profesores. Estudios pedagógicos, 42(4), 87-106.

Mercer, N. (2001). Palabras y mentes: cómo usamos el lenguaje para pensar juntos. Madrid: Planeta (GBS).

Merriam, S. B. \& Tisdell, E. (2009). Qualitative Research: A guide to design and implementation. San Francisco: Jossey-Bass.

Ministerio de Educación. (2013). A viva voz. Lectura en voz alta. Bibliotecas Escolares Cra. Santiago: Mineduc.

Ministerio de Desarrollo Social. (2016). Resultados Encuesta CASEN 2015. Santiago: MIDESO.

Molina-Valdés, E. P. y Haas-Prieto, V. (2018). Estudio de las interacciones profesor-alumno en la alfabetización de la geografía. Revista Colombiana de Educación, 75, 59-76.

Morrison, T. G., Jacobs, J. \& Swinyard, W. R. (1999). Do teachers who read personally use recommended practices in their classroom? Reading Research and Instruction, 38, 81-100.

Muñoz, C., Munita, F., Valenzuela, J. y Riquelme, E. (2018). Profesores que leen y enseñan a leer: El lugar de la lectura durante la formación. Revista ESPACIOS, 39(40), 32-42. 
Muñoz, C. \& Valenzuela, J. (2020). Demotivation in academic reading during teacher training. Journal of Research in Reading, 43(1), 41-56.

Muñoz, C., Valenzuela, J., Avendaño, C. y Núñez, C. (2016). Mejora en la motivación por la Lectura Académica: la mirada de estudiantes motivados. Ocnos: Revista de estudios sobre lectura, 15(1), 52-68.

OECD. (2014). Resultados PISA 2012 en foco: Lo que el alumnado saben a los 15 años de edad y lo que pueden hacer con lo que saben. Recuperado de. https://www.oecd.org/pisa/keyfindings/PISA2012_Overview_ESPFINAL.pdf

Orellana, P. \& Baldwin, P. (2018). Reading Motivation and Achievement Among Chilean Boys and Girls in Grades 3, 4, and 5: An Exploration of Results and Pedagogical Implications. In Reading Achievement and Motivation in Boys and Girls (pp. 115-133). Cham: Springer.

Orellana-García, P., Valenzuela, M. F. y Muñoz, K. (2018). Impacto de la lectura repetida interactiva en las habilidades verbales de preescolares de contextos vulnerables. Educación y Educadores 21(3), 409-432. DOI: 10.5294/edu.2018.21.3.3

Otto, B. (2008). Literacy development in early childhood. EE.UU.: Pearson.

Pontificia Universidad Católica de Chile. (2011). Alfabetización en establecimientos chilenos subvencionados: informe final. Santiago, Chile: Autor, Facultad de Educación. Recuperado de: http://www.uchile.cl/documentos/alfabetizacion-en-establecimientos-chilenos-subvencionadosministerio-de-educacion-puc-2011 84320 1 2030.pdf

Preiss, D. D., Calcagni, E., Espinoza, A. M., Gómez, D., Grau, V., Guzmán, V., Müller, M., Rámirez, F. y Volante, P. (2014). Buenas prácticas pedagógicas observadas en el aula de segundo ciclo básico en Chile. Psykhe, 23(2), 1-12.

Rockwell, E. (2001). La lectura como práctica cultural: conceptos para el estudio de los libros escolares. Educação e Pesquisa, 27(1), 11-26.

Rosenblatt, L. M. (1985). Viewpoints: Transaction versus interaction: A terminological rescue operation. Research in the Teaching of English, 19(1) 96-107.

Richardson, J. S. (2000). Read it Aloud! Using Literature in the Secondary Content Classroom. Newark, Delawere: International Reading Association.

Ruiz, M., Montenegro, M., Meneses, A. y Venegas, A. (2016). Oportunidades para aprender ciencias en el currículo chileno: contenidos y habilidades en educación primaria. Perfiles Educativos, XXXVIII(153), 16-33.

Sánchez, E, García, R. y Rosales, J. (2010). La lectura en el aula: Qué se hace, qué se debe hacer y qué se puede hacer. Barcelona: Graó.

Shanahan, T. \& Shanahan, C. (2012). What is disciplinary literacy and why does it matter? Topics in Language Disorders, 32(1), 7-18.

Street, J. C., \& Street, B. V. (1995). The schooling of literacy. In P. Murphy, M. Selinger, J. Bourne \& M. Briggs (Eds.), Subject Learning in the Primary Curriculum: Issues in English, Science and Mathematics (pp. 72-86). Londres: Routledge.

Swartz, S. L. (2010). Cada niño un lector: Estrategias innovadoras para enseñar a leer y escribir. Santiago: Ediciones UC. 
Tolchinsky, L. y Simó, R. (2001). Escribir y leer a través del currículum. Barcelona: ICE-Horsori.

Trostle, S. \& Donato, J. (2001). Storytelling in Emergent Literacity. Fostering Multiple Intelligences. DelmarThomson Learning, USA.

Unesco. (2009). Global Education Digest 2009. Comparing education statistics across the world. Montreal: Unesco.

Van den Broek, P., Kendeou, P., Kremer, K., Lynch, J. S., Butler, J., White, M. J. \& Lorch, E. P. (2005). Assessment of Comprehension Abilities in Young Children. Children's Reading. In S. G. Paris \& S. A. Stahl, Children's Reading Comprehension and Assessment (pp. 107-130). Mahwah, NJ: Lawrence Erlbaum Associates.

Valenzuela, J. P., Bellei, C. \& Ríos, D. (2014). Socioeconomic School Segregation in a Market-Oriented Educational System. The Case of Chile. Journal of Education Policy, 29(2), 217-241.

Wells, G. y Barberán, S. (2001). Indagación dialógica hacia una teoría y una práctica socioculturales de la educación. Barcelona: Paidós.

Winocur, R. (2015). Prácticas tradicionales y emergentes de lectoescritura en jóvenes universitarios. En N. García Canclini, V. Gerber, A. López, E. Nivón, C. Pérez, C. Pinochet Y R. Winocur, Hacia una antropología de los lectores. Ciudad de México: Paidós.

Zavala, V. (2011). La escritura académica y la agencia de los sujetos. Cuadernos Comillas, 1, 52-66.

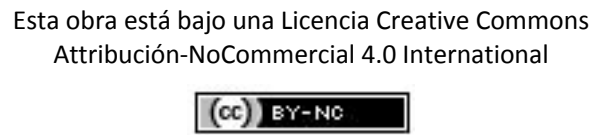

